\title{
ISOL TARGETS PREPARED WITH A NEW PAINT INFILTRATION COATING METHOD
}

\author{
Y. Kawai", G. D. Alton, J. O. Kiggans, D.W. Stracener, ORNL*, Oak Ridge, TN 37831, USA
}

\begin{abstract}
A new paint infiltration coating method has been developed for fabricating ISOL targets for radioactive ion beam applications. The technique has been shown to be inexpensive, fast, and almost universal for the uniform deposition of many refractory target materials onto the interior surfaces of complex geometry matrices, such as reticulated-vitreous-carbon-foam (RVCF). The process yields robust, highly permeable targets with fast diffusion and release properties. The use of compressed RVCF, coated with an optimum thickness of target material, reduces target lengths to practical values, while preserving high permeability. We have demonstrated the viability of the technique for coating RVCF compressed by factors of 6 and 10 with $\mathrm{WSi}_{2}$, to form targets for use at facilities such as the Rare Isotope Accelerator (RIA).
\end{abstract}

\section{INTRODUCTION}

Targets with fast diffusion and effusion properties are required in order to provide adequate intensity Radioactive Ion Beams (RIBs) for Isotope Separator OnLine (ISOL) research facilities such as the Holifield Radioactive Ion Beam Facility (HRIBF) [1]. Targets with fast diffusion release properties can be fabricated by infiltration deposition of controlled thicknesses of target materials onto the surfaces of complex, highly permeable matrices. Conventional infiltration deposition techniques are slow, expensive, and compound specific. We have developed an inexpensive, fast, and close to universal infiltration coating technique that overcomes these limitations [2,3]. Reticulated-vitreous-carbon-foam (RVCF) is an appropriate matrix for our application because of its high permeability. RVCF is available in compressed form in factors of $2,4,6$, and 10 in one direction, referred to as $2 \mathrm{x}, 4 \mathrm{x}, 6 \mathrm{x}$, and $10 \mathrm{xRVCF}$, respectively. Since matrices with higher compression ratios have larger surface-to-volume ratios, for a given target material coating thickness, ISOL targets can be shortened by using compressed RVCF. In this paper, we briefly describe the infiltration coating technique and demonstrate the viability of the technique for coating $6 x$ and $10 x \mathrm{RVCF}$ with $\mathrm{WSi}_{2}$. ( $\mathrm{WSi}_{2}$ is a candidate material for production of ${ }^{25} \mathrm{Al}$ and ${ }^{26 m} \mathrm{Al}$ via the respective ${ }^{28} \mathrm{Si}(\mathrm{p}, \alpha)^{25} \mathrm{Al}$ and ${ }^{28} \mathrm{Si}(\mathrm{d}, \alpha)^{26 \mathrm{~m}} \mathrm{Al}$ reactions. $)$

\section{TARGET FABRICATION}

Target fabrication begins by preparing the paint solution. Target material powders are reduced to $\phi \sim 1 \mu \mathrm{m}$ with an ultra-sonic milling device, then mixed into a YK

\footnotetext{
\#kawaiy@ornl.gov

*Managed by UT-Battelle, LLC, for the U.S. Department of Energy

under contract DE-AC05-0OR22725
}

binder [4] with a dispersant additive [5]. Disks of RVCF are then placed into a tapered holder attached to a chamber that can be evacuated by means of a roughing vacuum pump. Each disk is then uniformly covered with fluid by aspiration from an eyedropper while pulling air through the matrix through a leak valve connected to the roughing pump. After the fluid has been pulled through the sample, the sample is turned over and the excess fluid pulled back through the matrix in an effort to ensure coating uniformity.

Following coating, target disks are thermally treated in a vacuum furnace to decompose and drive off volatile components by gradually and linearly increasing the temperature to $\sim 800{ }^{\circ} \mathrm{C}$ over a 3 hour period. After this treatment, the coated target disks are raised to sintering temperatures $\left(\sim 1600{ }^{\circ} \mathrm{C}\right.$ for $\left.\mathrm{WSi}_{2}\right)$ under flowing $\mathrm{Ar}$.

\section{COATED TARGETS}

Coating thicknesses are found to increase almost linearly with volume $\%$ of target material over a practical range of thicknesses ( 0 to $\sim 16 \mu \mathrm{m}$ ), as shown in Fig. 1 . Coating thickness, $h$, given by

$$
h=\left(\Delta \mathrm{W} \cdot \rho_{0}\right) /\left(R_{\mathrm{SV}} \cdot \rho_{\mathrm{TM}} \cdot W_{\mathrm{BC}}\right)
$$

where $\Delta \mathrm{W}$ is the weight gain per disk after coating with target material followed by thermal treatment; $\rho_{0}=0.05$ $\mathrm{g} / \mathrm{cm}^{3}$ is the density of uncompressed RVCF; $R_{\mathrm{SV}}=32.2$ $\mathrm{cm}^{2} / \mathrm{cm}^{3}$ is the surface to volume ratio of uncompressed RVCF; $\rho_{\mathrm{TM}}$ is the bulk density of target material; and $W_{\mathrm{BC}}$ is the weight of compressed RVCF before coating.

Dimension control of the target material (thickness control for planar geometry deposits) is a critically important parameter since the speed of release must be fast with respect to the lifetime of the radioactive isotope of interest. The optimum thickness, $x$, for release of $\sim 70 \%$ of a species with lifetime $\tau_{1 / 2}$ from a planar geometry target is $[2,3]$

$$
x=\pi\left(D \tau_{1 / 2}\right)^{1 / 2}
$$

where $D$ is the diffusion coefficient of the product species in the target material. Since coating thicknesses increase almost linearly with volume \%, independent of target material, the desired coating thickness can be obtained simply by making a solution containing the appropriate mixture of target material.

Scanning electron micrographs (SEMs) of 6xRVCF before and after coating with $\sim 3.5 \mu \mathrm{m}$ of $\mathrm{WSi}_{2}$, using the infiltration coating technique, are displayed in Figure 2. As noted, the interior as well as the exterior surfaces of the disk are uniformly coated. Although the target solution was only aspirated from one side of the disk, no significant differences in the coating uniformity on the 
two sides were noted. The uniformity properties of the present samples are characteristic of those observed for $2 \mathrm{x}$ and $6 \mathrm{xRVCF}$ coated with different thicknesses of many other target materials $[2,3,6,7]$.

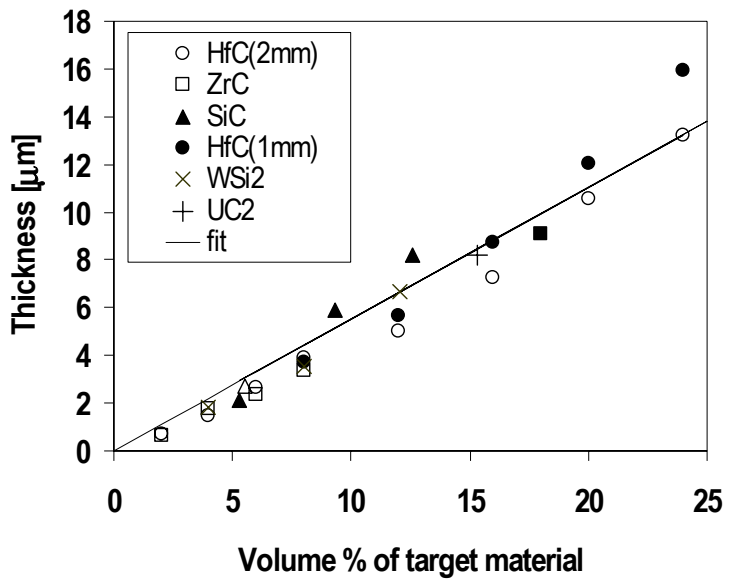

Figure 1: Correlation between volume mixture ratio of paint target material and target thickness coated onto 6xRVCF disks.

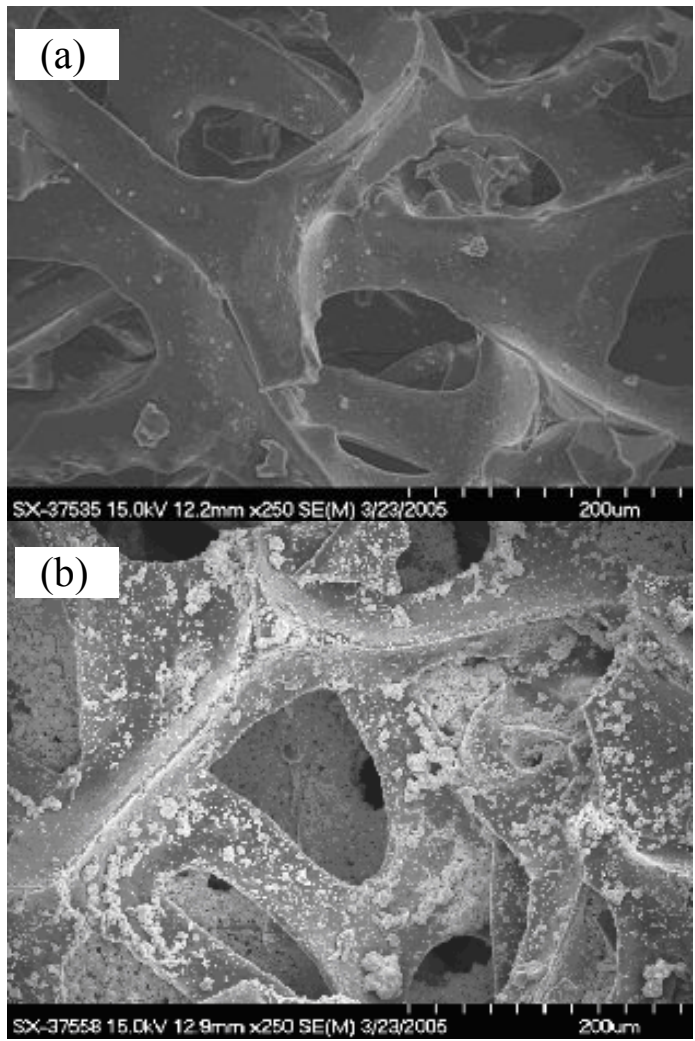

Figure 2: SEMs of (a) 6xRVCF without coating and (b) coated with $\sim 3.5 \mu \mathrm{m}$ of $\mathrm{WSi}_{2}$.

Figure 3 shows SEMs of 10xRVCF before and after coating with $\mathrm{WSi}_{2}$. As noted, $10 x \mathrm{xVCF}$ is less permeable than $6 \mathrm{xRVCF}$ and its structure much more disordered. The target solution was aspirated from the side of the disk shown in Fig. 3(b).

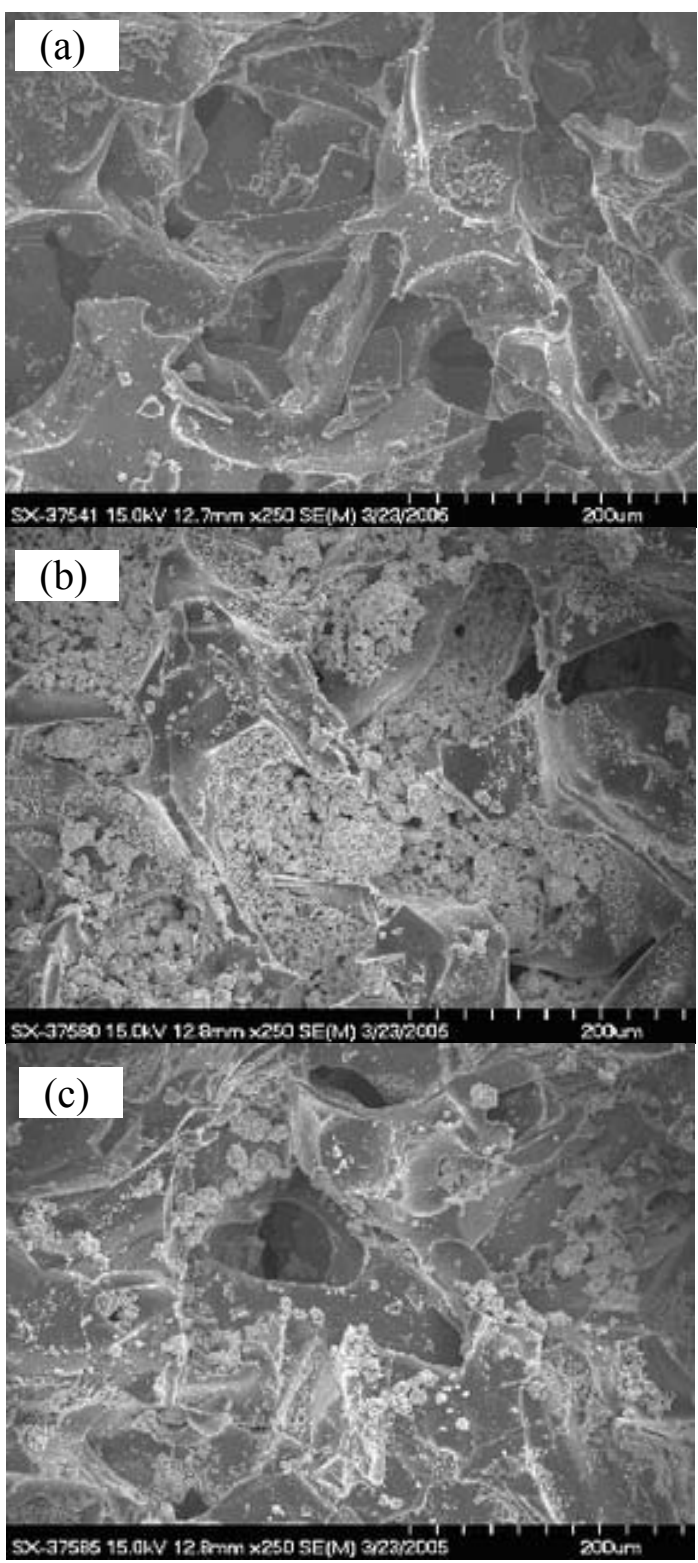

Figure 3: SEMs of (a) 10xRVCF without coating; (b) the side on which the paint solution was aspirated, and (c) the back side of the coated disk.

Because of the high viscosity of the paint solution and the limited pumping rate through the disk used in the current setup, the paint solution penetrates through the $10 \mathrm{xRVCF}$ at a very slow rate. Therefore, only very small amounts of paint solution can be aspirated at a time. As noted in Figs. 3b and 3c, the side from which the solution was aspirated appears to be more heavily coated. Apparently, the linear relation found between coating thicknesses and volume $\%$ of target material obtained for $6 x$ RVCF is no longer valid for 10xRVCF. However, the use of lower viscosity solvents and smaller diameter particles may ameliorate the non-uniformity problem.

The thickness estimations assume that the surfaces are uniformly coated with solid target material. In reality, 
there are openings between target material particles, as can be seen in Fig. 4. This is favorable feature for fast release of short-lived isotopes born within the solid material. Even for more densely coated surfaces, for example, as seen in the SEM displayed in Fig. 3 (b), ample openings exist between target material particles for atoms or molecules to escape following diffusion release.

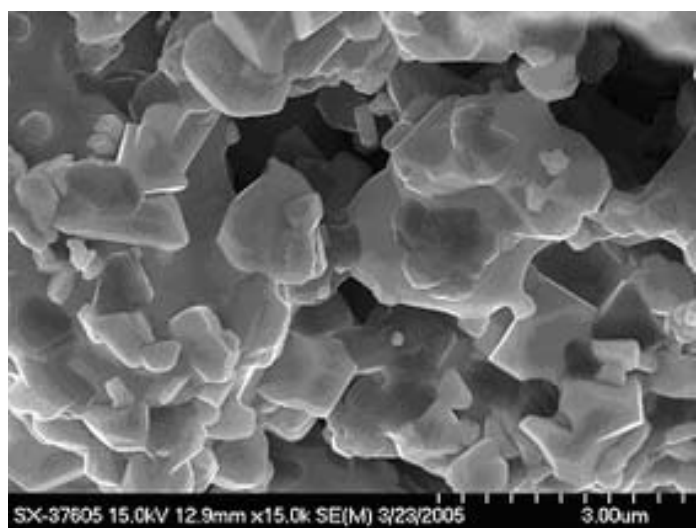

Figure 4: A high magnification $\mathrm{SEM}$ of a $\mathrm{WSi}_{2}$ deposit.

\section{CONCLUSIONS}

We have examined the viability of the paint infiltration technique for coating highly compressed $6 \times \mathrm{RVCF}$ and 10xRVCF for potential ISOL target applications. The paint coating technique can be utilized to fabricate relatively short, uniformly coated, optimum diffusionlength targets with the highly permeable, fast effusiveflow properties required of ISOL targets using $6 \times \mathrm{RVCF}$ as the coating matrix. However, further developments need to be made to improve the uniformity of depositions in more dense matrices such as 10xRVCF.

\section{ACKNOWLEDGEMENTS}

The authors are indebted to Ms. S.B. Waters and Ms. Dorothy Coffey for assistance in the Spex-milling and SEMs operations.

\section{REFERENCES}

[1] G.D. Alton and J.R. Beene, J. Phys. G, 24 (1998) 1347.

[2] J.-C. Bilheux, Ph.D. Thesis, Université de VersaillesSaint-Quentin, Versailles, France (2003).

[3] G.D. Alton, J.-C. Bilheux, A.D. McMillan, Nucl. Instrum. Methods A 521 (2004) 108.

[4] YK binder is a product of ZYP Coating Services, Oak Ridge, TN 37830, USA.

[5] Commercially available Menhaden Fish Oil.

[6] Y. Kawai, J.-C. Bilheux, D.W. Stracener, G.D.Alton, Appl. Rad. Isotopes (in print).

[7] Y. Kawai, J.-C. Bilheux, G.D.Alton, Nucl Instrum. Methods B (in print). 